INL/EXT-15-37497 Rev. 1

December 2015

\title{
Literature review report on atomistic modeling tools for FeCrAl alloys
}
Y. Zhang
D. Schwen
C. Jiang
E. Martinez

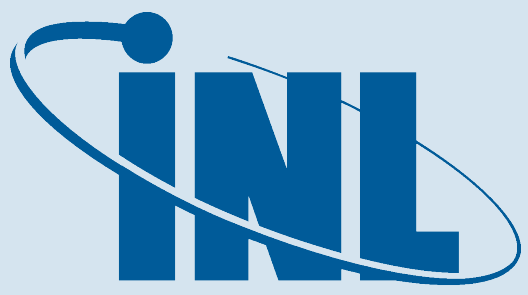

Idaho National Laboratory 


\section{NOTICE}

This information was prepared as an account of work sponsored by an agency of the U.S. Government. Neither the U.S. Government nor any agency thereof, nor any of their employees, makes any warranty, express or implied, or assumes any legal liability or responsibility for any third party's use, or the results of such use, of any information, apparatus, product, or process disclosed herein, or represents that its use by such third party would not infringe privately owned rights. The views expressed herein are not necessarily those of the U.S. Nuclear Regulatory Commission. 


\title{
Literature review report on atomistic modeling tools for
} FeCrAl alloys

\author{
Y. Zhang \\ D. Schwen \\ C. Jiang \\ E. Martinez* \\ December 2015
}

Idaho National Laboratory

Fuel Modeling and Simulation Department

Idaho Falls, Idaho 83415

*Los Alamos National Laboratory, Los Alamos, NM

Prepared for the

U.S. Department of Energy

Office of Nuclear Energy

Under U.S. Department of Energy-Idaho Operations Office

Contract DE-AC07-99ID13727 


\begin{abstract}
This report summarizes the literature review results on atomistic tools, particularly interatomic potentials used in molecular dynamics simulations, for FeCrAl ternary alloys. FeCrAl has recently been identified as a possible cladding concept for accident tolerant fuels for its superior corrosion resistance. Along with several other concepts, an initial evaluation and recommendation are desired for $\mathrm{FeCrAl}$ before it is used in realistic fuels. For this purpose, sufficient understanding on the in-reactor behavior of FeCrAl needs to be gained in a relatively short timeframe, and multiscale modeling and simulations have been selected as an efficient measure to supplement experiments and in-reactor testing for a better understanding of FeCrAl. Due to the limited knowledge on FeCrAl alloys the multiscale modeling approach relies on atomistic simulations to obtain the missing material parameters and properties. The purpose of this report is to identify relevant atomistic tools. It was noticed during the literature survey that no interatomic potentials are currently available for $\mathrm{FeCrAl}$. Here, we summarize the interatomic potentials available for $\mathrm{FeCr}$ alloys for possible molecular dynamics studies using $\mathrm{FeCr}$ alloys as surrogate materials. Other atomistic methods such as lattice kinetic Monte Carlo are also included in this report. A few possible research topics at the atomic scale are suggested based on the literature survey.
\end{abstract}




\section{Table of Contents}

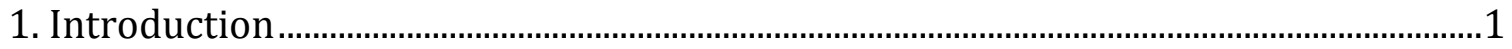

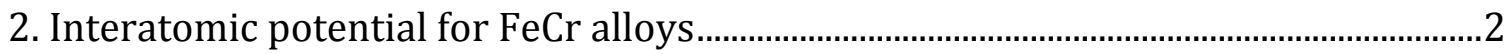

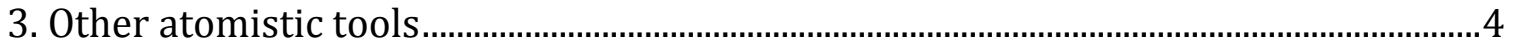

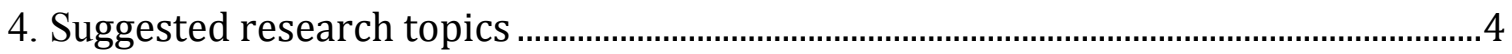

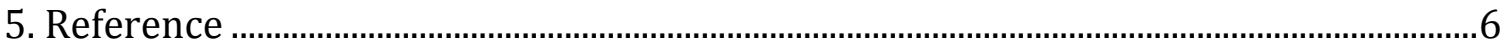




\section{Introduction}

Following the Fukushima Daiichi accident in 2011, the development of accident tolerant fuels (ATFs) has become a primary direction in nuclear fuel research and development for light water reactors (LWRs) [1]. Compared with the standard oxide fuel $\left(\mathrm{UO}_{2}\right.$ fuel with Zr-alloy cladding), the ATFs - including both fuels and claddings - are required to have better or at least comparable performance during normal operation, and better performance at accident scenarios such as loss of coolant accident (LOCA). The ATFs are expected to sustain a longer time than the oxide fuel under off-normal conditions and thus allow for enhanced safety margins. On the cladding side, several concepts have been proposed including FeCrAl alloys and SiC. For these concepts, full assessment of their in-reactor behavior is needed to give initial recommendations before licensing for commercialization.

Compared to the Zr-alloy claddings, FeCrAl alloys have superior resistance to water corrosion (oxidation) and higher mechanical strength at high temperatures [2]. The good corrosion resistance reduces the heat and hydrogen generation produced by reaction with coolant during severe accidents. The high mechanic strength enhances the burst margin of the cladding. The good corrosion resistance comes from the high $\mathrm{Cr}$ concentration and the formation of an aluminum-oxide layer at the surface. However, the beneficial properties of $\mathrm{FeCrAl}$ alloys are subject to change during neutron irradiation, which may induce (or enhance) phase separation, hardening, embrittlement, swelling, and compositional segregation. The properties and thus the performance of FeCrAl alloys depend on their microstructure and chemical composition. The FeCrAl alloys considered for ATF claddings usually have a high $\mathrm{Cr}$ content $(\sim 10-25 \mathrm{wt} \%)$ with up to $\sim 5 \mathrm{wt} \% \mathrm{Al}$. Within this $\mathrm{Cr}$ concentration range, $\mathrm{Cr}$ may precipitate into the $\mathrm{Cr}$-rich $\alpha$ phase at the cladding operating temperature $\left(\sim 300-400^{\circ} \mathrm{C}\right)$. This process can be drastically enhanced under neutron irradiation due to radiation-enhanced-diffusion. The formation of $\alpha$ phase particles hinders dislocation motion, leading to hardening and embrittlement. It also reduces the $\mathrm{Cr}$ concentration in the matrix and therefore potentially degrades the corrosion resistance. Neutron irradiation also produces lattice defects of either vacancy or interstitial type, which agglomerate to form extended defects such as dislocation loops and voids. These extended defects can also cause hardening and embrittlement by impeding dislocation motion. The formation of voids may also lead to swelling. Another factor that may degrade the superior properties of $\mathrm{FeCrAl}$ alloys is radiation-inducedsegregation (RIS). Due to the different interactions of the various solute elements with point defects (vacancies and interstitials), enrichment or depletion of solute elements may occur during irradiation at sinks such as grain boundaries, dislocations, and surfaces. This change in constituent distribution may result in degradation of the corrosion resistance and mechanical strength (e.g., intergranular fracture toughness). These irradiation effects have been extensively studied for $\mathrm{FeCr}$ alloys for their promising applications in fusion reactors. It's not clear how the addition of Al may change the behavior, calling for mechanistic understanding from both modeling and experimental sides. 
To supplement the experimental efforts in this area, the accident tolerant fuel high impact problem (ATF-HIP) under the Department of Energy (DOE) nuclear energy advanced modeling and simulation (NEAMS) program utilizes the multiscale tools developed under the same program to assess the performance of two leading ATF concepts: $\mathrm{U}_{3} \mathrm{Si}_{2}$ fuel and $\mathrm{FeCrAl}$ cladding [3]. The idea is to reduce the experimental efforts and to facilitate the evaluation by using NEAMS modeling tools, which are developed or under development for standard oxide fuels of LWRs. Under NEAMS, a microstructure based fuel performance modeling approach, the MOOSE-BISON-MARMOT (MBM) tool-kit, is currently being developed. The mesoscale tool MARMOT focuses on the microstructure evolution by taking information from the atomic scale and on the development of mechanistic materials models to be fed into BISON for engineering scale modeling. As a lot of mesoscale and engineering scale capabilities in MARMOT and BISON developed for oxide fuels can be borrowed for ATFs, the atomistic tools that supply necessary information into MARMOT need be identified.

Under NEAMS, atomistic simulations such as density functional theory (DFT) calculations and molecular dynamics (MD) simulations have been widely used to assist the development of MARMOT and BISON. Other atomistic methods such as lattice kinetic Monte Carlo (LKMC) and Metropolis Monte Carlo (MMC) are sometimes also involved. This report summarizes the literature review findings on atomistic tools that may be applied to $\mathrm{FeCrAl}$ alloys. In particular, we focus on interatomic potentials for $\mathrm{MD}$ simulations.

\section{Interatomic potential for $\mathrm{FeCr}$ alloys}

The research interest on $\mathrm{FeCrAl}$ alloys has remained limited until the recent identification of their potential application as ATF claddings. There has been no interatomic potential developed for $\mathrm{FeCrAl}$ alloys. On the other hand, owing to their promising application as structural materials for fusion reactors, $\mathrm{FeCr}$ alloys have been extensively studied from both modeling and experimental sides. For the similarities of $\mathrm{FeCr}$ and $\mathrm{FeCrAl}$ alloys in crystal structure and some other properties, $\mathrm{FeCr}$ alloys may be adopted as surrogate materials in modeling, with further consideration on the effects of $\mathrm{Al}$ addition.

In the past few decades multiple interatomic potentials with the embedded-atom-method (EAM) type have been developed, including the concentration-dependent-model potential (referred to as Caro2005) [4], and the two-band-model potentials (Olsson2003\&2006, Bonny2011, Eich2015) [5-8]. A detailed benchmark study of the first three potentials (Caro2005, Olsson2003\&2006) against DFT calculations was conducted by Klaver et al. in 2009 [9] on their performance on phase stability (heat of formation), lattice defects, $\mathrm{Cr}-\mathrm{Cr}$ interaction, and interaction between $\mathrm{Cr}$ and lattice defects and small clusters. It was concluded that these potentials treated the heat of formation of $\mathrm{FeCr}$ alloys fairly well at the Fe-rich side $(<12 \% \mathrm{Cr})$ but less satisfactorily beyond that. This is in agreement with a latter assessment which showed that the Olsson2003\&2006 and Bonny2011 potentials do not describe the $\alpha / \alpha$ miscibility gap well when the Cr concentration is high [8]. A similar drawback exits for the Caro2005 potential, which also performs well at the Fe-rich side. 
The Eich2015 potential fitted the mixing enthalpy to experimental data, and was shown to be able to reproduce well the $\alpha / \alpha$ miscibility gap against experimental data and the Calphad models [8]. It should be noted that many properties of $\mathrm{FeCr}$ alloys including the miscibility gap are related to magnetic effects, which are not explicitly considered in any of the above potentials.

The interaction between $\mathrm{Cr}$ atoms in solid solution is important for the nucleation of $\alpha$ ' phase. The interaction between $\mathrm{Cr}$ atoms in solid solution is repulsive, and the repulsion decreases with increasing distances. The Caro2005, Olsson2003\&2006 and Bonny2011 potentials have been shown $[7,9]$ to be able to reproduce reasonably well the trend given by DFT calculations on $\mathrm{Cr}-\mathrm{Cr}$ interaction. However, the Olsson2003\&2006 potentials failed to predict the drop in repulsion from the first nearest neighbor (1NN) to $2 \mathrm{NN}$ distances. It's not clear how the Eich2015 potential behaves on this.

The interaction of $\mathrm{Cr}$ atoms with point defects and small defect clusters is import for $\mathrm{Cr}$ transport and precipitation of $\alpha$ phase under irradiation. In comparison to DFT calculations, the Olsson2003\&2006 and Bonny2011 potentials were found to give better results on the stability of interstitials with various configurations, and the interaction between them and $\mathrm{Cr}$ atoms [7,9]. Therefore they are so far the best choices to study the microstructure evolution of $\mathrm{FeCr}$ alloys under irradiation.

The kinetics of $\alpha$ phase precipitation is determined by the diffusion of $\mathrm{Cr}$ atoms. Under thermal aging the diffusion is mainly mediated by vacancies due to the high formation energy of interstitial. Under irradiation, transport via interstitial mechanisms becomes important, too. So far, only the migration barriers of $\mathrm{Cr}$ via vacancy exchange have been benchmarked against DFT calculations, and the results given by the Bonny2011 potential matched the best with DFT calculations, followed by the Olsson2006 potentials [7]

The core structure of the $1 / 2<111>$ screw dislocation (in bulk Fe and $\mathrm{Cr}$ ) and its stable glide in a $\{110\}$ plane in bulk Fe were also simulated using the Olsson2006 and Bonny 2011 potentials. The latter was found to be the only one that can predict the compact core structure of the $1 / 2<111>$ screw dislocation, and was deemed the most suitable potential to study the interaction between $\mathrm{Cr}$ atoms and screw dislocations [7]. The behavior of screw dislocations is critical to understand the mechanical deformation of bcc ferritic steels.

The above information indicates that so far the Eich 2015 potential may be the best choice for the phase separation in $\mathrm{FeCr}$ alloys at thermal equilibrium. Other potentials are also good for this purpose while dealing with Fe-rich alloys, particularly when the $\mathrm{Cr}$ concentration is below the solubility limit. For instance, the Caro potential was used to predict the critical size for $\alpha$ phase nucleation [10]. In situations when precipitation kinetics is of interest and/or irradiation is involved, the Bonny2011 potential seems to be a good choice. It's also the best choice for mechanical behavior so far. The Olsson2006 and Bonny2011 potentials have also been shown suitable for microstructure evolution under irradiation. However, to simulate the defect production by displacement cascades as produced by high-energy neutrons, the screened Coulomb interaction at short range 
needs to be included. The Eich2015 potential is new, and more assessment is needed to fully elucidate its applicability in areas other than thermodynamic phase separation.

\section{Other atomistic tools}

In addition to MD, other atomistic tools such as DFT and LKMC have also been used to study the behavior of FeCr alloys. DFT calculations have widely been used to explore the fundamental properties of $\mathrm{FeCr}$ alloys, and to fit and assess interatomic potentials for these alloys. LKMC simulations have been carried out to study RIS in FeCr alloys [11]. However, applications of these methods in the much more complex ternary FeCrAl alloys are rare. Recently, an LKMC model has been developed for the phase separation of FeCrAl alloys under thermal aging [12]. In this model, the Bonny2006 potential was used to describe the interaction between $\mathrm{Fe}$ and $\mathrm{Cr}$, with the $\mathrm{Fe}-\mathrm{Al}, \mathrm{Cr}-\mathrm{Al}$ and $\mathrm{Al}-\mathrm{Al}$ interactions described by a rigid lattice potential fitted to DFT results. The model was used to simulate the thermal aging of Fe-10Cr-(4-8)Al alloys, and the results obtained were in good agreements with experimental measurements. This suggests that the LKMC model is suitable for $\alpha$ precipitation in FeCrAl alloys under thermal aging. The set of parameters describing the interatomic interactions may also be used in MMC simulations for the phase separation at thermal equilibrium since the strain mismatch between $\alpha$ and $\alpha$ phases is negligible.

\section{Suggested research topics}

$\mathrm{Fe}-\mathrm{Cr}-\mathrm{Al}$ is a pretty complex ternary system. Given that the ATF-HIP is a 3-years project, to develop an interatomic potential for the Fe-Cr-Al system may not be feasible to meet the timeline. With the atomistic tools currently available, some atomic scale research topics are suggested as listed below.

\section{- $\alpha$ ' precipitation under thermal aging}

With the set of parameters for LKMC modeling, the precipitation of $\alpha$ phase under thermal aging with various chemical compositions at different temperatures can be explored. The phase separation at thermal equilibrium can be obtained using MMC simulations with the same set of parameters. The information obtained from LKMC simulations, such as the size and density of $\alpha$ precipitates, will be used to estimate the hardening.

\section{- Dislocation - a' precipitates interaction in FeCr alloys to evaluate hardening}

Due to the similarities of $\mathrm{FeCr}$ and FeCrAl alloys, particularly when the Al concentration is low, $\mathrm{FeCr}$ alloys can be taken as surrogate materials to investigate the hardening induced by $\alpha$ precipitates. MD simulations can be used to study the interaction between dislocations and $\alpha$ ' precipitates of various sizes. As shown above, the Bonny 2011 potential gives the correct core structure of screw dislocations, which are crucial for the 
mechanical deformation of bcc ferritic alloys. Therefore, the Bonny 2011 potential can be utilized for this purpose. The effect of added $\mathrm{Al}$ can be assessed once a FeCrAl potential becomes available. Results from MD simulations, in supplementation to the available data in literature [13], will provide input for upper scale modeling such as viscoplasticity modeling for hardening and creep.

\section{- Radiation induced segregation in FeCrAl alloys}

Radiation induced segregation in ternary alloys has been formulated in theoretical models [14]. Applying the previous theory to RIS requires a set of parameters on the effective diffusivities of alloying elements via vacancy mechanisms and those via interstitial mechanisms. To study RIS in FeCrAl, DFT calculations are needed to elucidate the diffusion mechanisms of alloying elements via vacancy and interstitial mechanisms. The results from DFT calculations can be taken into the existing theoretical framework to evaluate RIS at various temperatures and dose rates. To allow for more complexity, an LKMC model (a set of pairwise bonds) can be trained by DFT calculations [15] on the formation energies of vacancy, interstitial, and their clusters. The additions of $\mathrm{Cr}$ and $\mathrm{Al}$ can be accounted for by including the heat of mixing and the defect configurations involving alloying elements in the fitting. Note that in LKMC, in general no off-lattice configurations are allowed. Interstitials are treated as atoms of a certain element type on the lattice. The modeling results on RIS will indicate if the constituent distribution in FeCrAl alloys will be changed substantially during neutron irradiation. The LKMC modeling can be done based on the model for $\mathrm{FeCr}$ binary alloys [10] with extensions to FeCrAl ternary alloys. The redistribution of alloying elements may induce phase instability and degrade the corrosion resistance of FeCrAl alloys.

\section{- Phase field model development in MARMOT}

The parameters used in the LKMC model [12] can also be used to assist the development of a phase field model in MARMOT for FeCrAl alloys. In literature these are thermodynamic data for $\mathrm{FeCrAl}$ alloys regarding the phase stability [16]. However, it's not clear how well that free energy model predicts the miscibility gap between $\alpha$ and $\alpha$ ' phases. MMC simulations with the set of parameters in literature [12] can be used to refine the free energy model [16] (if needed), which can then be implemented into MARMOT for phase field simulations of phase separation at large time and spatial scales. The phase field model can also be used to model swelling in FeCrAl alloys induced by voids or helium bubbles. 


\section{Reference}

1. S. Bragg-Sitton, Development of advanced accident-tolerant fuels for commercial LWRs, Nuclear News, March 2014, 83.

2. K. A. Terrani, S. J. Zinkle, L. L. Snead, Advanced oxidation-resistant iron-based alloys for LWR fuel cladding, Journal of Nuclear Materials 448 (2014) 420-435.

3. K. A. Gamble, J. D. Hales, J. Yu, Y. Zhang, D. Andersson, A. Patra, W. Wen, C. Tomé, M. Baskes, E. Martinez, C. R. Stanek, Y. Miao, B.Ye, G. L. Hofman, A. M. Yacout, Multiscale Multiphysics Developments for Accident Tolerant Fuel Concepts, INL/EXT-15-36761, September 2015.

4. A. Caro, D. A. Crowson, M. Caro, Classical Many-Body Potential for Concentrated Alloys and the Inversion of Order in Iron-Chromium Alloys, PRL 95 (2005), 075702.

5. P. Olsson, I. A. Abrikosov, L. Vitos, J Wallenius, Ab initio formation energies of Fe-Cr alloys, Journal of Nuclear Materials 321 (2003), 84.

6. P. Olsson, I. A. Abrikosov, J. Wallenius, Electronic origin of the anomalous stability of Fe-rich bcc Fe-Cr alloys, Phys. Rev. B 73 (2006), 104416.

7. G. Bonny, R. C. Pasianot, D. Terentyev, L. Malerba, Iron chromium potential to model high-chromium ferritic alloys, Philosophical Magazine 91 (2011), 17241746.

8. S. M. Eich, D. Beinke, G. Schmitz, Embedded-atom potential for an accurate thermodynamic description of the iron-chromium system, Computational Materials Science 104 (2015), 185-192.

9. T. P. C. Klaver, G. Bonny, P. Olsson, D. Terentyev, Benchmarking FeCr empirical potentials against density functional theory data, Modelling Simul. Mater. Sci. Eng. 18 (2010), 075004.

10. D. Schwen, E. Martinez, A. Caro, On the analytic calculation of critical size for alpha prime precipitation in FeCr, Journal of Nuclear Materials 439 (2013), 180184.

11. O. Senninger, F Soisson, E. Martínez, M. Nastar, C. C. Fu, Y. Brechet, Modeling radiation induced segregation in iron-chromium alloys, Acta Materialia 103 (2016), 1-11.

12. J. Ejenstam, M. Thuvander, P. Olsson, F. Rave, P. Szakalos, Microstructural stability of $\mathrm{Fe}-\mathrm{Cr}-\mathrm{Al}$ alloys at $450-550{ }^{\circ} \mathrm{C}$, Journal of Nuclear Materials 457 (2015), 291-297.

13. D. Terentyev, G. Bonny, C. Domain, R. C. Pasianot, Interaction of a $1 / 2<111>$ screw dislocation with $\mathrm{Cr}$ precipitates in bcc Fe studied by molecular dynamics, Phys. Rev. B 81 (2010), 214106.

14. G. Was, T. Allen, Radiation-induced-segregation in Multicomponent alloys: Effect of Particle Type, Materials Characterization 32 (1994), 239-255.

15. Piochaud, Jean-Baptiste, Modelling of radiation induced segregation in austenitic Fe alloys at the atomistic level, PhD Thesis, 2013.

16. M. H. G. Jacobs, R. Schmid-Fetzer, T. Markus, V. Motalov, G. Borchardt, K. H. Spitzer, Thermodynamics and diffusion in ternary $\mathrm{Fe}-\mathrm{Al}-\mathrm{Cr}$ alloys, Part I:

Thermodynamic modeling, Intermetallics 16 (2008), 995-1005. 